\title{
Problemas da Fiziologia Nervoza
}

PROF. H. LAUGIER

Com intensa curiozidade fui entrevistar o Prof. Laugier que actualmente dá em S. Paulo um curso de fiziologia nervoza, promovido pelo Instituto Tecnico FrancoPaulista. Espirito vibrante, inteligencia perfectibilizada pelo estudo e pela cultura, pesquizador incansavel dos problemas da fiziologia nervoza, o Prof. Laugier é grandemente conceituado no mundo científico.

Mantive com êle longa palestra. Manifestou com entuziasmo a sua admiracão por S. Paulo. Falou com idealismo sobre o intercambio intelectual entre os estudantes da Franca e os do Brazil. Referiu-se sobre a nobre finalidade da Caza do Estudante Estrangeiro em Paris.

Adiante publicamos o que o Prof. Laugier escreveu para a Revista de Medicina.

\section{- QUE PENSEZ VOUS DES ECHANGES INTELLE- CTUELS FRANCO-BRESILIENS?}

- Je les crois appéles à se développer tous les jours davantage; et je souhaite de tout coeur qu'ils deviennent de plus en plus nombreux et de plus en plus frequents. J'ai toujours eté un partisan ardent des relations intellectuelles, artistiques e scientifiques entre tous Wes pays; mais particulierment entre deux pays comme le Brésil et la France qui ont un fonds de culture commune aussi riche et qui sont faits pour se comprendre et pous s'aimer. Le travail des hommes de science, dans tous les domains, est souvent silencieux et obscur; il n'en est pas moins vrai que c'est lui qui, plus que tout autre fait progresser l'humanité et l'achemine vers des jours meilleurs, non seulement au point de vue materiel, mais au point de vue moral. Or, rien ne facilite le travail scientifique comme le contact personnel en- 
tre les hommes que s'occupent des mêmes disciplines, et qui, en divers points du monde, travaillent à conquerir chaque jour de nouveaux terrains sur l'inconnu.

C'est pourquoi j'ai eté très hereux, lorsque, gráce aux organisatéurs de l'Institut Franco-Pauliste, et à l'aimable hospitalité de l'Etat de S. Paulo, la faculté m'a eté donnée de venir dans se pays admirer la genereuse ardeur de votre action dans tous les domaines intellectuels scientifiques, medicaux et techniques et d'y apporter ma modeste contribution; je suis très sensible à l'honneur que m'a eté fait lorsque l'on m'a demandé de professer á la Faculté de Medecine, un bref enseignement sur une serie de methodes nouvelles, etablie au cours des vingt derniéres annèes au Laboratoire de Physiologie generale de" la Sorbonne. Dés l'arrivée j'ai eté infiniment touché par l'acueil qui m'a eté fait; et exetrememint frappé par l'interet reél, l'attentiton soutenue, que les etudiants ont apporté à l'étude de ses questions, souvent un peu arides, mais d'une portée generale considerable. C'est dans une atmosphère de sympathie et de grande ardeur au travail que j'ai commencé et continué mes conferecences. Je ne saurais dire combien j'en ai eté profondement touché.

M:on voeu le plus cher serait que ses échanges puissent se poursuivre non seulement entre professeurs, mais entre etudiants; se serait un profit incalculabre pour nos deux grandes patries si chaque année un nombre important d'etudiants pouvaint passer l'Atlantique et aller les uns en France, les autres au Brésil, y nourrir á la fois des relations intellectuelles et des relations d'amitié. Esprons que ce beau rêve daviendra une realité dans un avenir prochain.

- QUELLES SONT LES APPLICATIONS DE LA CHRONAXIE; EST CE QUE LES METHODES FONDEESS SUR LA CHRONAXIE MODIFIENT E SIMPLIFIENT LES PROCEDES ACTUELS DE L'ELECTRODIAGNOSTIC?

- La chronaxie est une caracteristique objective, très penetrante, très sensible, de l'excitabilité des tissus, fibres nerveuses, nerfs, cordons nerveux du systeme cerebro spinal; du sympathique, e muscles. Elle est facile à determiner, par une technique simple et la methode 


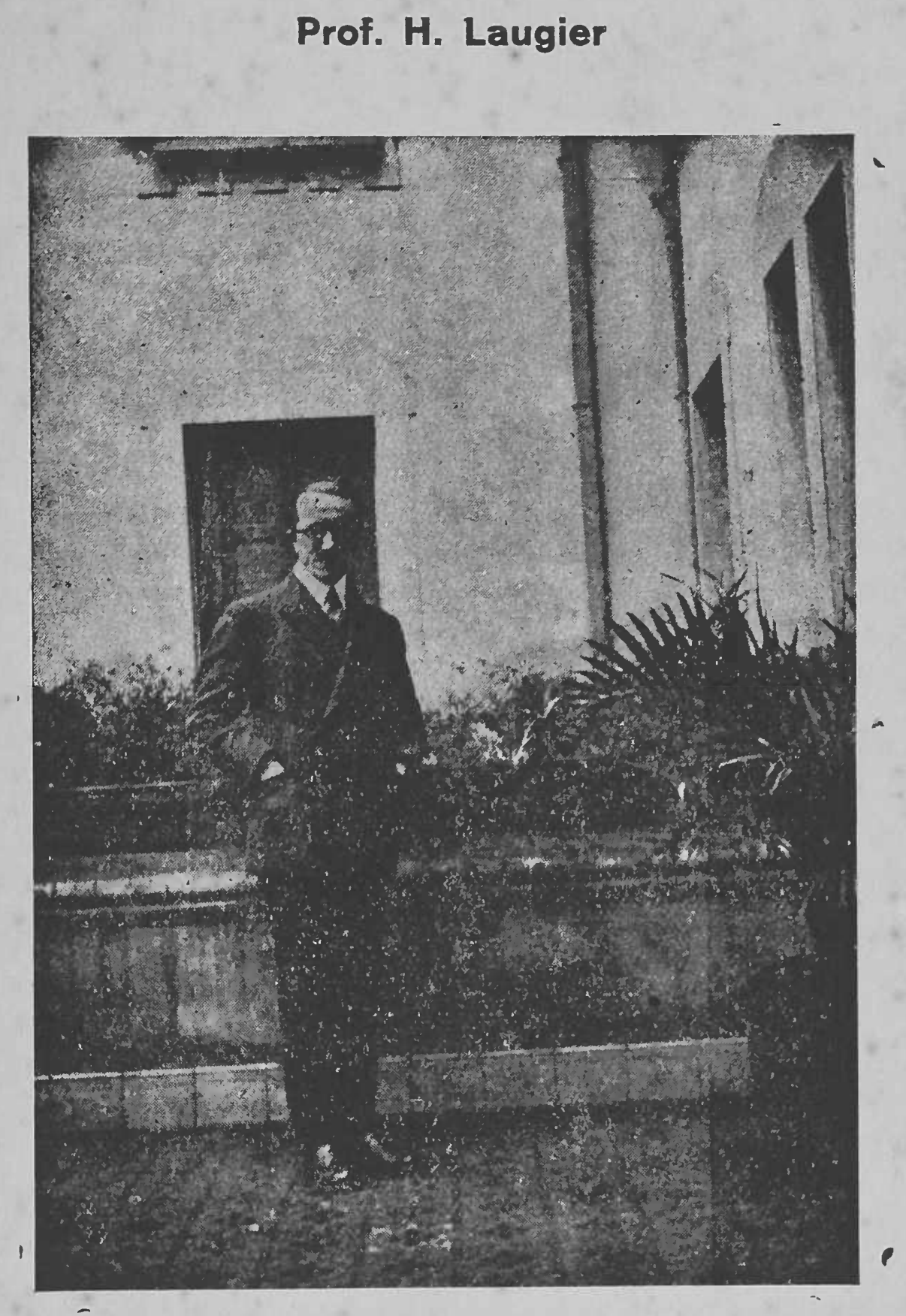

5. 5. póza especialmente para a "Reyista de Medicina" 

donne un chiffre précis. En l'utilisant, en realise immèdiatament dans le domaine de la connaissance du systeme nerveux, et des applications qui en resultent, tous les progrés que l'on realise dans un domaine quel toonque lorsque l'on satisfait au "besoin de mesure", et lorsque l'on substitue à une simple appreciation qualitative des faits ,une mesure quantitative precise.

Progrés dans le domaine physiologique - En etudiant la chromaxie d'un tissu on attend dum meme coup toute les caracteristiques essențielles de son excitabilité. Ainsi, la sensibilité d'un tissu aux courants est fonction de la chromaxie. De meme sa faculté de sommation; de meme, les carateristiques essentielles de l'influx nerveux (vitesse de propagation, frequence e durée des ondes de negativité dont il est constitué). - En outre, une notion capitale nouvelle résulte des 'travaux de L. e M. Lapicque d'une part; de C. Veil, d'autre part; orsque deux tissus exitables se font sente, et que l'excitation se transmet d'un tissu à l'autre, les conditions de passage sont determínées par le rapport des chromaxies des deux tissus. Lorsque ces rapports physiologiques des valeurs des chromaxies sont perturbé, l'excitation éprouve à passer d'un tissu à l'autre une defficulté que peut arreter completement la transmission.

C'est ainsi que peuvent etre expliquées les actions du curare, et de la strychnine, par exemple. On conçoit ainsi que les phenoménes d'aiquillage qui sont d'une importance primordiale dans le fon"ctionnement du systeme nerveux central se trouvent conditionnés par les valeurs des chromaxies des differents neurones.

Progrés du pharmacologie et en toxiçologie - La chromaxie varie chaque fois que l'etat du sissu consideré varie; s'est un reactif trés fidèle et trés sensible, qui permet de chiffrer l'action d'un poison, d'un medicament, d'un extrait biologique, que l'etude au moyen de la tchronaxie de l'action des venins, et même sans doute des seruns antti-venimeux, permettra de faire des progrés importants dans la connaissance des lois de leur action, et dans leur etalonnage. C'est un domaine de choix pour les travailleurs scientifiques de S. Paulo, qui disposent sur ce point d'un materiel expérimentel unique au monde.

Progrés en electrodiagnostic - La chronaxie se modifie considerablement au cours des degenerescences; la sensibilté de ses radia- 
tions au cours des divers etats pathologiques permet de reveler et de chiffrer avec precision des alterations du système neuro musculaire finfiniment plus faibles que celles qu'atteignent les methodes de l'elejetrodiagnostic classique. Les premier resultats observés ayec une mekhode simplifié par Bourguignon et Laugier, puis avec la technique plus precise des decharges de condensatoun, par Bourgnignon, sont extremement suggestifs. Il n'est pas douteux que dans un avenir rapproché, l'étude de la chronłaxie penetrera definitivement en pathologie . et constituera le centre même de l'Electrodiagnostic.

Prof. H. Langier.

(Professor de Fiziologia da Sorboune. França).

\section{PHARMACIA LANGE PREÇOS ESPECIAES PARA MEDICOS E ESTUDAN- TES DE MEDICINA}

Pedidos por Telephone - Central 2223

Rua Vergueiro, 10 - S. Paulo 